fibrosis. Nine age- and sex- matched post-mortem myocardial samples served as histological controls.

Results When compared to controls, the myocardium of patients with severe AS had reduced vessel density, total quantity of SMA + ve and CD31 +ve, in addition to the expected increase in fibrosis. There was absence of CD31 +ve endothelium in SMA +ve arterioles, indicating endothelial loss. (Figure 1) Importantly, patients with an aortic valve area (AVA) $\leq 0.8 \mathrm{~cm}^{2}$ had greater endothelial loss compared to those with an AVA $>0.8$ and $\leq 1.0 \mathrm{~cm}^{2} \quad(1.34 \%$ $\pm 0.44 \%$ vs $2.84 \pm 1.03 \%, p=0.006$ ), and endothelial loss also correlated with myocardial perfusion reserve index (MPRI), $\mathrm{r}=0.66, \mathrm{p}=0.019$. MPRI improved significantly post AVR (from $0.95 \pm 0.17$ to $1.50 \pm 0.43, \mathrm{p}=0.018$ ).

Conclusion In severe AS, there is microvascular rarefaction and loss of endothelium, which is more pronounced in patients with the most severe aortic valve narrowing. This appears to be an underlying mechanism for reduced myocardial perfusion reserve, which may be reversible post AVR.

\section{INTRACARDIAC THROMBI IN CARDIAC AMYLOIDOSIS, A COMMON FINDING}

${ }^{1}$ Tushar Kotecha, ${ }^{2}$ Esther Gonzalez-Lopez, ${ }^{1}$ Andrej Corovic, 'Sarah Anderson, 'Liza Chacko, ${ }^{1}$ James Brown, ${ }^{1}$ Dan S Knight, ${ }^{3}$ A John Baksi, ${ }^{4}$ James C Moon, ${ }^{5}$ Peter Kellman, ${ }^{2}$ Pablo Garcia-Pavia, ${ }^{1}$ Julian D Gillmore, ${ }^{1}$ Philip N Hawkins, 'Marianna Fontana. ${ }^{1}$ Royal Free Hospital, London, UK; ${ }^{2}$ University Hospital Puerta de Hierro Majadahonda, Madrid, Spain; ${ }^{3}$ Royal Brompton Hospital, London, UK; ${ }^{4}$ Barts Health NHS Trust, London, UK; ${ }^{5}$ National Institutes of Health, National Heart, Lung and Blood Institute, Bethesda, USA

\subsection{6/heartjnl-2019-BSCMR.21}

Background Cardiac amyloidosis (CA) has been associated with a high prevalence of intracardiac thrombi, but this was reported in small cohorts of high risk patients (with a clinical indication for transoesophageal echocardiography). It is therefore not known whether such observations are applicable to the general CA population.

Purpose To assess the prevalence of intracardiac thrombi in patients with CA.

Methods 324 consecutive patients with CA were studied prospectively using a standard CMR protocol at $1.5 \mathrm{~T}$, including early and late gadolinium imaging and T1 mapping. Early

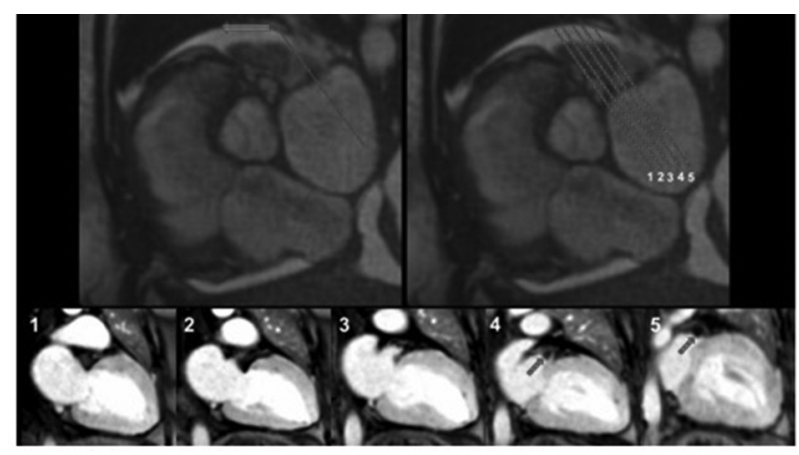

Abstract 21 Figure 1 Acquisition of stack through the LAA. Early gadolinium images of the LAA (bottom row) acquired using a $5 \mathrm{~mm}$ contiguous stack through the LAA (top row) and an inversion time of $440 \mathrm{~ms}$ to confirm the presence or absence of thrombus vs normal pectinate muscle. The thrombus in the left atrial appendage can only be visualised in the last two images (red arrows in panel 4 and 5) and could have been missed with the acquisition of only one image gadolinium images (segmented imaging, trigger 2) of the left atrial appendage (LAA) were acquired using a $5 \mathrm{~mm}$ contiguous stack and a TI of $440 \mathrm{~ms}$.

Results The study participants comprised 155 with light chain CA (AL), 166 with transthyretin amyloidosis (ATTR), 2 with Apo A-I, and 1 with Apo A-IV CA. The prevalence of intracardiac thrombi was $5.2 \%$ in AL, 7.2\% in ATTR; 6.2\% overall. $90 \%$ of thrombi were in the LAA. This was higher when there was atrial fibrillation $(9.1 \% \mathrm{AL}, 14.3 \%$ ATTR) but intracardiac thrombi were also present in sinus rhythm (SR) $3.1 \%$ (4.5\% AL, 1.1\% ATTR). In all patients with AF the thrombi were present despite long term anticoagulation. The presence of intracardiac thrombi was associated with a greater degree of systolic dysfunction and myocardial amyloid infiltration (higher native T1 and ECV).

Conclusions The prevalence of intracardiac thrombi in CA and $\mathrm{AF}$ is high despite long term anticoagulation, with significant thrombus prevalence even in SR, meriting vigilance for intracardiac thrombi in all. CMR with early gadolinium imaging of the LAA is a valuable screening tool for thrombi in the LAA and should be routine part of the clinical protocol when amyloidosis is suspected. Current guidelines for electrical cardioversion after prolonged anticoagulation without screening for thrombus in the LAA should not be applied to patients with CA.

\section{IMPAIRED STRESS-INDUCED OXYGENATION IN HYPERTROPHIC CARDIOMYOPATHY IS ASSOCIATED WITH AN INCREASED RISK OF VENTRICULAR ARRHYTHMIA}

${ }^{1}$ Betty Raman, ${ }^{1}$ Masliza Mahmod, ${ }^{1}$ Kenneth Chan, 'Sanjay Sivalokanathan, ${ }^{2}$ Theodoros D Karamitsos, ${ }^{3}$ Joseph Selvanayagam, ${ }^{1}$ Rina Ariga, ${ }^{1}$ Aaron T Hess, ${ }^{1}$ Elizabeth M Tunnidiffe, ${ }^{1}$ Hugh Watkins, ${ }^{1}$ Stefan Neubauer*. 'Oxford Centre for Clinical Magnetic Resonance Research, Division of Cardiovascular Medicine, Radcliffe Department of Medicine, University of Oxford, Oxford, UK; ${ }^{2}$ First Department of Cardiology, Aristotle University of Thessaloniki, AHEPA Hospital, Thessaloniki, Greece; ${ }^{3}$ Department of Cardiovascular Medicine, Flinders Medical Centre, Bedford Park, Adelaide, Australia

\subsection{6/heartjnl-2019-BSCMR.22}

Background Myocardial ischaemia is believed to promote fatal life-threatening ventricular arrhythmias in hypertrophic cardiomyopathy (HCM). Oxygen sensitive cardiac magnetic resonance (CMR) or blood oxygen level dependent (BOLD) imaging can detect blunted myocardial oxygenation during vasodilator stress in HCM. Whether or not impairment in stress oxygenation is associated with ventricular arrythmia risk is unknown.

Objectives To investigate the relationship between blunted stress oxygenation and ventricular arrhythmia in HCM and examine the determinants of stress oxygenation in HCM.

Methods 103 genotyped HCM patients and 32 (age, gender and body mass index matched) healthy controls underwent adenosine stress BOLD, stress first pass perfusion imaging and late gadolinium imaging (LGE) to assess stress oxygenation, myocardial perfusion reserve index (MPRI), and fibrosis burden respectively. Stress oxygenation response (BOLD $\Delta$ SI) was estimated as a relative increase in oxygen sensitive BOLD signal intensity from rest to peak vasodilator stress. All HCM patients had 24-holter monitoring to assess for ventricular tachycardia ( $\geq 3$ beats, $\geq 120$ beats per minute).

Results As expected, both MPRI $(1.5 \pm 0.4 \quad v \quad 2.0 \pm 0.3$, $\mathrm{p}<0.0001)$ and stress oxygenation $(9.1 \% \pm 4.1 \% \quad v \quad 17.0 \%$ 